\title{
VARIATIONS DURING AGEING IN THE THREE-DIMENSIONAL ANATOMICAL ARRANGEMENT OF FASCICLES WITHIN THE EQUINE SUPERFICIAL DIGITAL FLEXOR TENDON
}

\author{
O.J. Ali ${ }^{1,2}$, E.J. Comerford ${ }^{1,3,4}$, P.D. Clegg ${ }^{1,3,4}$ and E.G. Canty-Laird ${ }^{1,4, *}$ \\ ${ }^{1}$ Department of Musculoskeletal Biology, Institute of Ageing and Chronic Disease, University of \\ Liverpool, William Henry Duncan Building, 6 West Derby Street, Liverpool, L7 8TX, UK. \\ ${ }^{2}$ Current affiliation: Department of Surgery and Theriogenology, College of Veterinary Medicine, \\ University of Sulaimani, Sulaimani, Iraq. \\ ${ }^{3}$ Institute of Veterinary Science, Leahurst Campus, University of Liverpool, Chester High Road, Neston, \\ CH64 7TE, UK. \\ ${ }^{4}$ The MRC-Arthritis Research UK Centre for Integrated research into Musculoskeletal Ageing (CIMA), \\ Liverpool, L7 8TX, UK.
}

\begin{abstract}
Tendons are constructed from collagenous fascicles separated by endotenon/interfascicular matrix (IFM). Tendons may be specialised for precision movement or to store energy during locomotion and for the latter the elasticity of the endotenon/IFM is particularly important. The equine superficial digital flexor tendon (SDFT) is a dedicated energy-storing tendon with a similar function to the human Achilles tendon. Classical anatomical descriptions portray fascicles as longitudinally arranged distinct anatomical structures. In the present study, using three-dimensional reconstruction from whole tissue slices and histological sections, the fascicles of the equine SDFT were found to adopt a complex interweaved arrangement. Fascicles were found to fully and partially converge and diverge within the tendon and fascicle bundles were observed. Fascicle morphology was not homogenous with narrowing, broadening and twisted fascicles observed in addition to relatively straight fascicles. The number of fascicle bundles observed in cross-section increased from the proximal to the distal end of the tendon, whilst the number of fascicles decreased with age in the proximal region. Fascicular patterns were not similar between the left and right limbs, across different regions or at different ages. A decrease in thickness of the endotenon/IFM between fascicles with age was found in the distal tendon region. The results provide a rationale for considering fascicular organisation when diagnosing and treating tendon injuries, for bioengineering tendon and when modelling tendon function.
\end{abstract}

Keywords: Tendon, fascicles, 3D reconstruction, endotenon, interfascicular matrix, ageing, equine, SDFT.

*Address for correspondence: Dr Elizabeth Gail Canty-Laird, Department of Musculoskeletal Biology, Institute of Ageing and Chronic Disease, University of Liverpool, William Henry Duncan Building, 6 West Derby Street, Liverpool, L7 8TX.

Tel: +441517946026_Ｅmail: elizabeth.laird@liverpool.ac.uk

\section{Introduction}

Tendons function to transmit force from muscle to bone. Energy-storing tendons act as biological springs to decrease the energetic cost of locomotion (Alexander, 1991), whilst positional tendons facilitate the precise control of movement (Ward et al., 2006). The superficial digital flexor tendon (SDFT) in the horse and the Achilles tendon in man are extensible energy-storing tendons that are susceptible to agerelated degeneration and non-contact injury. Tendon or ligament injury accounts for around $50 \%$ of musculoskeletal injuries in racehorses (Thorpe et al., 2010a). Tendon injury is the most common reason for retirement in racing Thoroughbreds and age is a risk factor for tendon injury in horses (Kasashima et al.,
2004; Perkins et al., 2005). In humans, rupture of the Achilles occurs in $0.1 \%$ of the population (Hess, 2010) and accounts for 3-17 per 100,000 hospital admissions in Europe (Clayton and Court-Brown, 2008; Maffulli et al., 1999; Nyyssonen et al., 2008). The risk of certain tendon injuries in man also increases with age, with lower limb tendons such as the Achilles being more commonly injured in middle age, and upper limb tendons such as the rotator cuff undergoing pronounced deterioration in older age (Clayton and Court-Brown, 2008). Damaged tendons heal by producing an inferior quality scar-like tissue that leads to reduced strength and increased likelihood of re-rupture.

Tendons are constructed from collagenous fascicles separated by interfascicular endotenon. The 
endotenon/interfascicular matrix (IFM) is a loose vascularised connective tissue situated between collagenous fascicles (Kannus, 2000). The endotenon/ IFM facilitates sliding between tendon fascicles, is more elastic in energy-storing tendons and shows functional and structural deterioration with age (Thorpe et al., 2015a; Thorpe et al., 2012; Thorpe et al., $2013 b)$. The relatively cellular endotenon/IFM has a more complex protein composition and a higher rate of turnover than the fascicles (Thorpe et al., 2016a; Thorpe et al., 2016b). The endotenon/IFM is present during foetal development at which stage it appears to be thicker than in adult tendons (Russo et al., 2015).

Due to differences in methodologies in studies spanning several decades as well as variations in the size and specialisation of tendons from different locations and species, there are inconsistencies in the terminology used for each level of the hierarchical collagenous structure of tendon. A recent proposition has been made for a consistent size-based naming scheme, which we have adopted for this study (Handsfield et al., 2016). Within fascicles, tendon fibroblasts initially synthesise short parallel collagen fibrils (Birk and Trelstad, 1986; Birk et al., 1989; Canty et al., 2004), which are thought to mature by accretion of collagen molecules at specific sites on the fibril surface, as well as by end-to-end fusion (Kalson et al., 2015). Hence collagen fibrils increase in length and diameter. Estimates of fibril length, derived from counts of the number of fibril ends in serial electron microscopy images, range upwards of 1-14 mm (Craig et al., 1989; Kalson et al., 2015; Svensson et al., 2017). Based on a paucity of collagen fibril ends in tendon, it is suggested that end-to-end fibril fusion may be limited only by tendon length, such that collagen fibrils can act as the primary force transducers in mature tendon (Provenzano and Vanderby, 2006). Continuous fibrils are observed in the short mouse stapedius tendon; however, structural fibril continuity is not required for mechanical continuity if fibrils exceed a critical length (estimated as $1 \mathrm{~mm}$ ), due to surface interactions becoming stronger than the fibril itself (Svensson et al., 2017). Collagen fibrils themselves are bundled into helical fibres between cell processes within fascicles (Kalson et al., 2015), within which such surface interactions would take place. Bundles of fascicles are observed in the equine SDFT, which is derived solely from one muscle attachment, whilst the human Achilles is divided into subtendons, each arising from different muscle bellies and previously termed 'fascicles' or 'fascicle bundles' (Handsfield et al., 2016).

Fascicles are usually considered to be distinct entities that run from one end of the tendon to the other (Edwards, 1946; Kastelic et al., 1978). In the human supraspinatus tendon no interdigitation is observed but around one-fifth of fascicles/fascicle bundles converge between the muscle and the bone (Fallon et al., 2002). However, extraction of tendon fascicle (termed fibre bundle) structure by ultrasound image processing indicates that fascicles may not be continuous in the equine SDFT (Meghoufel et al., 2010). The presence of discontinuous fascicles, separated by endotenon/interfascicular matrix would prevent force transmission through fascicular collagen fibrils alone and would have important implications for tendon biomechanics. Understanding the 3D topography of tendon fascicles is important for understanding the molecular mechanisms of force transmission and elasticity in tendon and to create valid models of tendon mechanics. In the present study we analysed the 3D architecture of fascicles and interfascicular matrix in the extensible energy-storing equine SDFT and determined how fascicle numbers and endotenon/IFM thickness changed with age at different levels of the tendon.

\section{Materials and Methods}

\section{Tissue samples}

SDFT samples were harvested from horses of different ages, euthanised for reasons other than orthopaedic disease or injury. Anonymised samples were obtained either from a commercial equine abattoir, or from horses that were euthanased at the Philip Leverhulme Equine Hospital, University of Liverpool, with the owner's informed consent for research. The study was assessed and approved by the University of Liverpool's Veterinary School Research Ethics Committee (VREC 214). Material derived from 13 horses was used for preparation of whole tissue slices and material derived from 26 horses for histology (Table 1).

\section{Preparation and imaging of whole tissue slices}

Dissected SDFT samples, representing the whole metacarpal or metatarsal length extending from the carpometacarpal to the metacarpophalangeal joints, were wrapped in foil and stored at $-20{ }^{\circ} \mathrm{C}$. Frozen SDFT samples were sectioned transversely in a palmar-dorsal or plantar-dorsal direction over a dissecting wooden cutting board using a post-mortem knife. The thickness of the cut sections was 2-3 mm. All tendon sections were placed on wet tissue paper, with the proximal aspect facing upward and arranged in a straight line from proximal to distal. Sections were hydrated for 10-15 min in a flat container under slow-dripping tap water. With hydration, the endotenon/IFM bulged and appeared as whiteoutlined structures that could easily be observed between the fascicles. Sections were transferred individually onto a transparent flat glass plate adjacent to a calibrated ruler and photographed sequentially without flash (Canon EOS 5D Mark III, 1000 ISO-speed with a Canon EF 100mm F2 .8L Macro IS USM Lens) at a distance of approximately $200 \mathrm{~mm}$. The resulting JPEG images were cropped and converted to greyscale, using Paint Shop Pro X4 Ultimate, by selecting channel mixer ( $-40 \%$ red, $-40 \%$ green, $-40 \%$ blue and $110-120 \%$ contrast) followed by a fade correction of 50 to enhance image 
Table 1. Details of samples used for the study. Abbreviations: LF; left forelimb, RF; right forelimb, LH; left hind limb, $\mathrm{RH}$; right hind limb, NS; not shown (area).

\begin{tabular}{|c|c|c|c|c|}
\hline Age & Leg & Paired & Processing method & Resulting data presented in Figure: \\
\hline 4 & LF & $\mathrm{Y}$ & Tissue slices & $2 \mathrm{e}, 3,4, \mathrm{NS}$ \\
\hline 4 & RF & $\mathrm{Y}$ & Tissue slices & $2 \mathrm{e}, 3,4, \mathrm{NS}$ \\
\hline 5 & RF & $\mathrm{N}$ & Tissue slices & $2 \mathrm{e}, 4, \mathrm{NS}$ \\
\hline 6 & LF & $\mathrm{Y}$ & Tissue slices & $2 \mathrm{a}, 2 \mathrm{e}, 3,4, \mathrm{NS}$ \\
\hline 6 & RF & $\mathrm{Y}$ & Tissue slices & $2 \mathrm{e}, 3,4, \mathrm{NS}$ \\
\hline 7 & LF & $\mathrm{N}$ & Tissue slices & $2 \mathrm{e}, 4, \mathrm{NS}$ \\
\hline 8 & LF & $\mathrm{N}$ & Tissue slices & $2 \mathrm{e}, 4,5$ \\
\hline 8 & LF & $\mathrm{N}$ & Tissue slices & 4,5 \\
\hline 8 & RT & $\mathrm{N}$ & Tissue slices & 4,5 \\
\hline 11 & RF & $\mathrm{N}$ & Tissue slices & $4, \mathrm{NS}$ \\
\hline 12 & LF & $\mathrm{N}$ & Tissue slices & $2 \mathrm{~b}, 2 \mathrm{c}, 2 \mathrm{~d}, 2 \mathrm{e}, 4$ \\
\hline 17 & LF & $\mathrm{N}$ & Tissue slices & $2 \mathrm{e}, 4, \mathrm{NS}$ \\
\hline 18 & $\mathrm{RH}$ & $\mathrm{Y}$ & Tissue slices & $2 \mathrm{e}, 3,4, \mathrm{NS}$ \\
\hline 18 & LH & $\mathrm{Y}$ & Tissue slices & $2 \mathrm{e}, 3,4, \mathrm{NS}$ \\
\hline 20 & $\mathrm{RH}$ & $\mathrm{N}$ & Tissue slices & $4, \mathrm{NS}$ \\
\hline 21 & LF & $\mathrm{Y}$ & Tissue slices & $2 \mathrm{e}, 3,4,5, \mathrm{NS}$ \\
\hline 21 & RF & $Y$ & Tissue slices & $2 \mathrm{e}, 3,4, \mathrm{NS}$ \\
\hline Foetal & RF & $\mathrm{N}$ & Histology & 8 \\
\hline 1 & RF & $\mathrm{N}$ & Histology & 6 \\
\hline 1 & RF & $\mathrm{N}$ & Histology & 7 \\
\hline 1 & RF & $\mathrm{N}$ & Histology & 8 \\
\hline 3 & RF & $\mathrm{N}$ & Histology & 6 \\
\hline 3 & RF & $\mathrm{N}$ & Histology & 6 \\
\hline 3 & LF & $\mathrm{N}$ & Histology & 6 \\
\hline 4 & RF & $\mathrm{N}$ & Histology & 6 \\
\hline 5 & RF & $\mathrm{N}$ & Histology & 6 \\
\hline 7 & RF & $\mathrm{N}$ & Histology & 6 \\
\hline 7 & RF & $\mathrm{N}$ & Histology & 6 \\
\hline 7 & LF & $\mathrm{N}$ & Histology & 6 \\
\hline 9 & RF & $\mathrm{N}$ & Histology & 6 \\
\hline 9 & RF & $\mathrm{N}$ & Histology & 6 \\
\hline 9 & RF & $\mathrm{N}$ & Histology & 6 \\
\hline 9 & RF & $\mathrm{N}$ & Histology & 6 \\
\hline 9 & RF & $\mathrm{N}$ & Histology & 6 \\
\hline 9 & RF & $\mathrm{N}$ & Histology & 8 \\
\hline 14 & LF & $\mathrm{N}$ & Histology & 6 \\
\hline 14 & LF & $\mathrm{N}$ & Histology & 6 \\
\hline 14 & RF & $\mathrm{N}$ & Histology & 6 \\
\hline 17 & RF & $\mathrm{N}$ & Histology & 6 \\
\hline 18 & RF & $\mathrm{N}$ & Histology & 6 \\
\hline 18 & LF & $\mathrm{N}$ & Histology & 6 \\
\hline 19 & RF & $\mathrm{N}$ & Histology & 6 \\
\hline 20 & RF & $\mathrm{N}$ & Histology & 6 \\
\hline
\end{tabular}

clarity. The Enhance Photo toolbar was then used to adjust Local Tone Mapping (Strength: 100, Block Size: 50) followed by a High Pass Sharpen in Hard Light blend mode. Fade correction was repeated when required to further enhance image clarity. A specific crop contour size was applied to all sections equally and all cropped photos saved as TIFF images for compatibility with IMOD software (Kremer et al., 1996) used for 3D reconstruction.

\section{Processing and analysis of images derived from whole tissue slices}

Greyscale JPEG images were imported into ImageJ (Version 1.49) (Schneider et al., 2012). The 3D surface plot of each image was adjusted by selecting the following parameters; Filled, Original Colors, Grid Size: 512, Perspective: 0.1-0.25, z-Ratio - xy-Ratio, Invert, Scale: 1.40-1.70, z-Scale: 4.00-4.10, Max: $65-75 \%$, Min: 0-10 \%, with the optimum value for fascicular discrimination being primarily dependent on the Max and Min values. Fascicles and fascicle bundles were categorised on the basis of apparent groupings of two or more fascicles, approximate size and endotenon/IFM thickness (Fig 1A), and then counted in the proximal, mid-metacarpal and distal regions. Endotenon/IFM thickness was measured at 100 points on ruler-calibrated greyscale images from the proximal, middle and distal regions for 

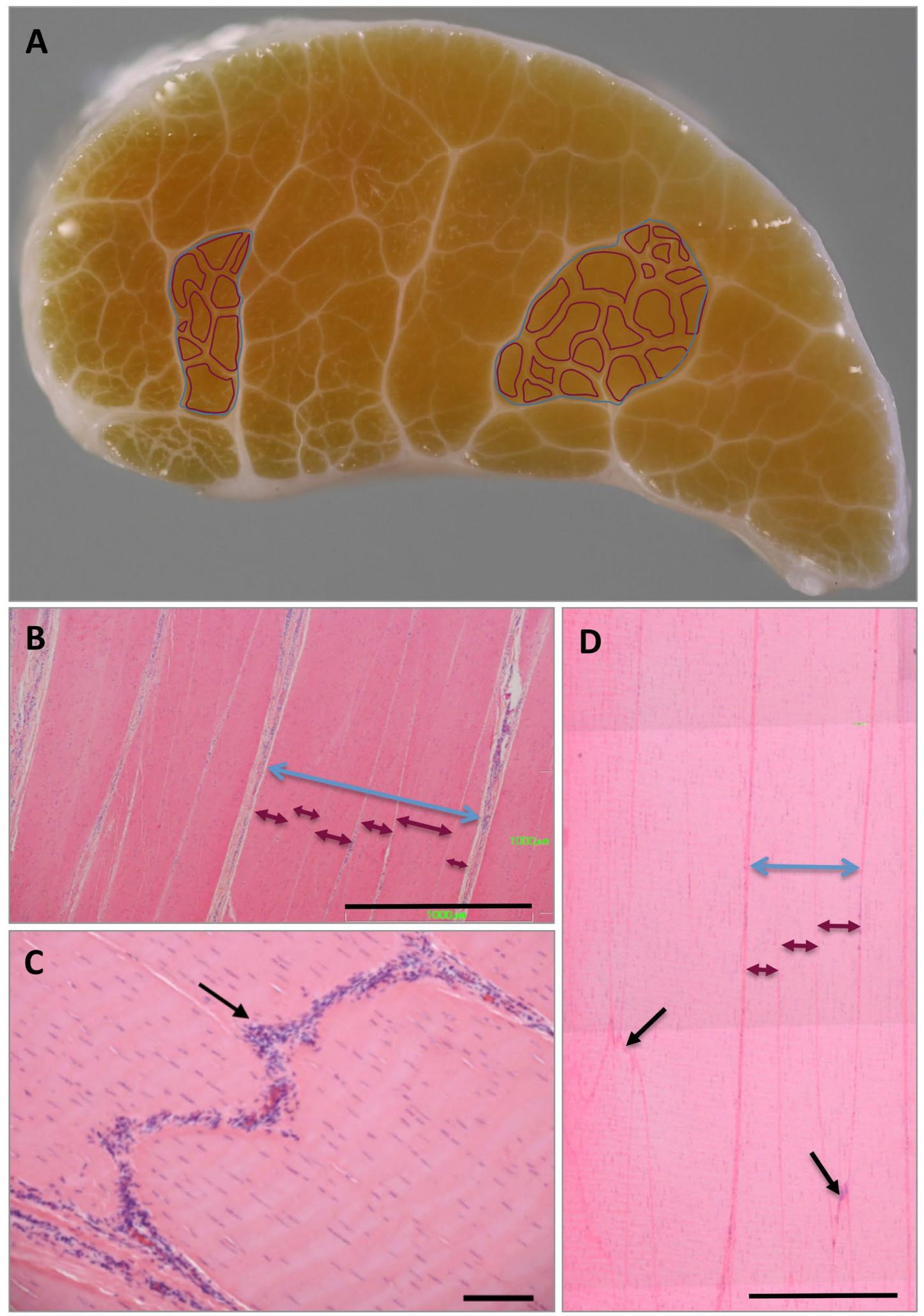

Fig. 1. Appearance of the superficial digital flexor tendon in whole tissue slices and histological sections. A: Single image of one transverse tissue slice before conversion to greyscale. Two fascicles bundles are indicated (blue) together with the individual fascicles (magenta). Outlines are shrunk to allow visualisation of the endotenon/IFM (white). The collagenous fascicles appear yellow/brown in colour images. B: Single colour image of a longitudinal histological section stained with H\&E. Fascicle bundles and fascicles are indicated using the same colour scheme in A. Endotendon/IFM can be seen between the fascicles and bundles. Scale bar $=1 \mathrm{~mm}$. C: Histological section stained with H\&E containing transversely-orientated endotenon/IFM (black arrow). Scale bar $=100 \mu \mathrm{m}$. D: Part of a collage of H\&E stained histological sections used for 3D reconstruction. Bundles (blue arrow), fascicles (magenta arrows) and apparent fascicle discontinuities (black arrow) are indicated. Scale bar $=1 \mathrm{~mm}$. 
fascicles and fascicle bundles using the 'Set Scale' and 'Measure' tools in ImageJ. Cross-junctional areas of the endotendon/IFM were excluded from the analysis as they formed irregular stellate structures.

\section{Preparation, imaging and analysis of histological sections}

Samples (Table 1) approximately $10 \mathrm{~mm}$ in length were taken from the tendon core in the proximal, middle and distal metacarpal regions. Specimens were fixed in $4 \%$ buffered formaldehyde solution, $\mathrm{pH} 7.4$ at room temperature, dehydrated with an ethanol series and then paraffin-embedded before sectioning longitudinally in the sagittal plane. Sections were collected onto poly-L-lysine-coated slides (VWR International, Lutterworth, UK) and stained with haematoxylin and eosin (H\&E) (TCS Biosciences, Buckingham, UK) (Fig. 1 B,C). Sections were imaged using an Eclipse 80i microscope (Nikon, Japan) and endotenon/IFM thickness measured at 60 points on ruler-calibrated images.

Samples for serial sectioning were fixed in $4 \%$ paraformaldehyde (Sigma-Aldrich, Gillingham, UK) for $24 \mathrm{~h}$, dehydrated with an ethanol series and then paraffin-embedded for sectioning longitudinally. Sections were taken in the frontal plane for foetal and one-year-old samples (Table 1). The larger nine-yearold sample (Table 1) was divided sagittally before embedding and only one half of an approximately $10 \mathrm{~mm}$ long block was then sectioned longitudinally on the sagittal plane. The tissues were initially trimmed by taking $100 \mu \mathrm{m}$ sections and then twenty $5 \mu \mathrm{m}$ longitudinal sections collected for analysis. For one additional one-year-old sample, the whole depth of a trimmed tissue block from the mid-metacarpal region was cut into 500 sections. Sectioning orientation and the location of any missing sections was recorded. Serial sections were collected on to poly-L-lysine slides and stained with H\&E. All sections were stained at the same time to obtain the same staining density and contrast. Several images were taken using the $4 \times$ objective so that the entire surface was captured. All captured photos were collated and converted to one image using Inkscape (www.inkscape.org). All image collages were cropped using Paint Shop Pro x4 Ultimate and converted to Tiff images (Fig 1D) for compatibility with IMOD.

\section{$3 \mathrm{D}$ reconstruction}

IMOD, a suite of image processing, modelling and display programs (Kremer et al., 1996), was used for $3 \mathrm{D}$ reconstruction of endotenon/IFM and fascicles from whole tissue slices and histological sections. Briefly, TIFF images were automatically converted to an image stack using the IMOD program 'tif2mrc'. The image stack was then manually aligned using the IMOD program 'midas'. Isosurface reconstructions of the endotenon/IFM were created from '.mrc' image stacks using the Isosurface tool, which uses pixel-level thresholding, within the IMOD program ' $3 \mathrm{dmod}$ '. Isosurface parameters were adjusted manually to delineate the endotenon/IFM similarly to that observed in the original images. 3D reconstruction of fascicles and fascicle bundles was carried out manually within ' $3 \mathrm{dmod}$ ' by outlining individual fascicles or fascicle bundles, with contours from a single object, in sequential images within the image stack. IMOD was also used to determine the cross-sectional area of tissue slices by outlining the entire slice. For 3D reconstruction, the z-distance was set based on image magnification and slice thickness, in order to separate contours in 3D, and contours then meshed to create a 3D object for each fascicle. Each fascicle or bundle was assigned a specific colour. 3D reconstruction was carried out using images derived from whole tissue slices of the mid-metacarpal region. Reconstructions of fascicles derived from the central portion of images from 3D slices of the mid-metacarpal region (Table 1) were viewed using 'Model View' and tracked using the 'ZaP Window' in IMOD. The number of observed divergence, convergence, partial convergence and re-splitting events were recorded. Divergence was considered to be where fascicles or bundles divided into two or more separate branches. Convergence was considered to be where two or more fascicles or bundles merged together into a single fascicle. Partial convergence was considered to be where regions of two adjacent fascicles were observed to join and separate intermittently within the reconstruction. Resplitting was where a divergence event was observed after a convergence event. 3D reconstruction of all fascicles within a $10 \mathrm{~mm}$ length of SDFT from the mid-metacarpal region of a one-year old horse (Table 1) was carried out using alternate sections from a series of 500 serial histological images. Smaller reconstructions of foetal, one-year and nine-year old tissue were carried using out using 20 sequential serial images.

\section{Statistical analysis}

Data analysis was performed using SigmaPlot 13. Normally-distributed data (Shapiro-Wilk test) were analysed by one-way or two-way ANOVA with a Holm-Sidak post-hoc test. Data failing the ShapiroWilk normality test were analysed using KruskalWallis one-way ANOVA on ranks with a post-hoc Tukey test. Pearson correlation was used to derive correlation coefficients and $p$-values for age-related alterations in fascicle numbers and endotenon/IFM thickness. Significance was set at $p \leq 0.05$.

\section{Results}

\section{Single fascicle bundles were not continuous throughout the entire tendon}

In preliminary studies, viewing images stacks derived from slices through an entire tendon, it was found to be not possible to track individual fascicle bundles from the proximal to the distal end of the 

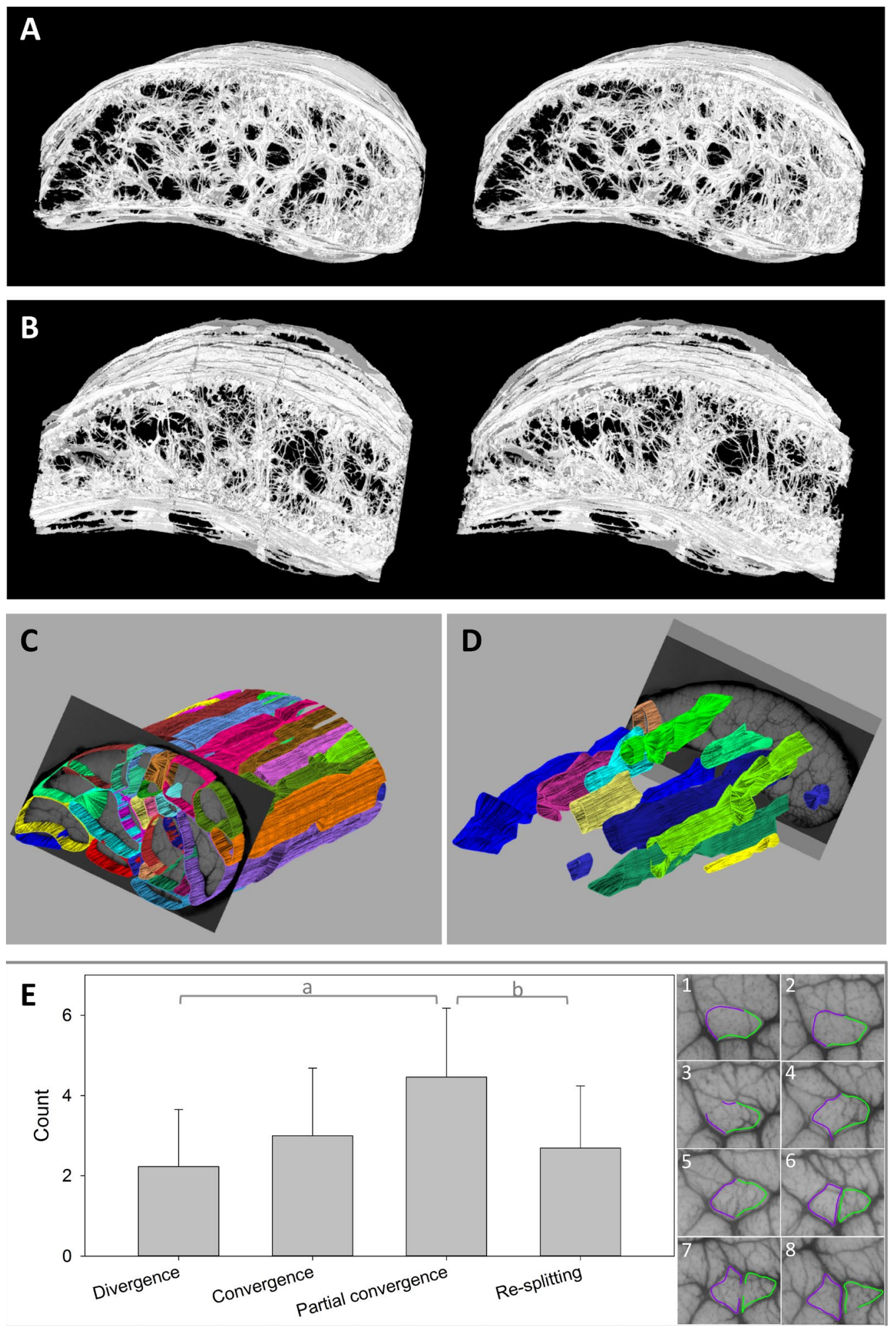

Fig. 2. 3D fascicular structure derived from whole tissue slices. A \& B: Stereopair view of an isosurface of the endotenon/IFM and epitenon (white) derived from the mid-metacarpal region of six-year-old (A) and 12-year-old (B) tendon. C \& D: 3D reconstruction of fascicle bundles in a $65 \mathrm{~mm}$ length of the mid-metacarpal region of 12 year old tendon. All reconstructed fascicle bundles are included in $\mathbf{C}$, whilst $\mathbf{D}$ shows selected bundles from the 3D reconstruction. E: Measurements of the occurrence of fascicle divergence, convergence, partial convergence and re-splitting in single fascicle bundles tracked through the mid-metacarpal region of 13 samples ranging from 4 to 21 -years-old (left). ${ }^{\mathrm{a}} p=0.005,{ }^{\mathrm{b}} p=0.034$ by one-way ANOVA with HolmSidak post-hoc test. An example of partial convergence as illustrated for two individual fascicles is shown (right). Fascicle outlines are shrunk to allow visualisation of the endotenon/IFM. In panel 6 the fascicles are separate, partially converge in 7 and separate again in 8 where the green fascicle is partially converged with another adjacent fascicle. Panel 3 shows the purple fascicle partially converging with another fascicle due to thinning of the endotenon/IFM. 
tendon. This indicated that the tendon was not simply made up of several separate well-defined parallel fascicles. Focusing on the mid-metacarpal region of the tendon (Video 1) allowed an isosurface model of the endotendon/IFM to be created (Fig. 2A-B). The endotenon/IFM was seen to comprise an intricate network and was continuous with the surrounding epitenon. By then outlining individual fascicle bundles in the mid-metacarpal region, a 3D model of all fascicle bundles was created (Fig. 2C). Some fascicle bundles were present in all tissue slices but some did not continue as separate entities within the image stack, primarily due to convergence or divergence (Fig. 2D). Single fascicles were found to undergo several divergence/convergence events with the frequency of partial convergence being higher than that of divergence or re-splitting (Fig. 2E).

\section{Tendons showed limited left-right asymmetry}

Comparing images of whole tendon slices in the proximal, mid-metacarpal and distal regions of the left and right forelimbs of the same horse from samples aged 4, 6, 18 and 21 years old (Fig. 3) indicated that the fascicular structure was neither identical nor were they mirror images of one another. Some minor similarities, for example in the location of the widest part of the endotenon/IFM adjacent to the epitenon, could occasionally be seen. $p$

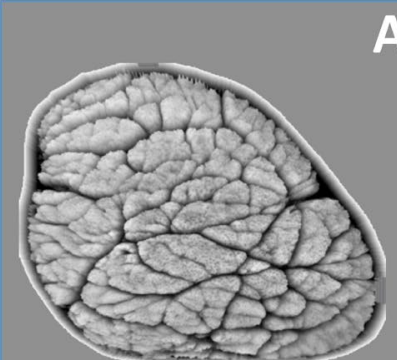

$\mathbf{A}$

$\mathrm{m}$
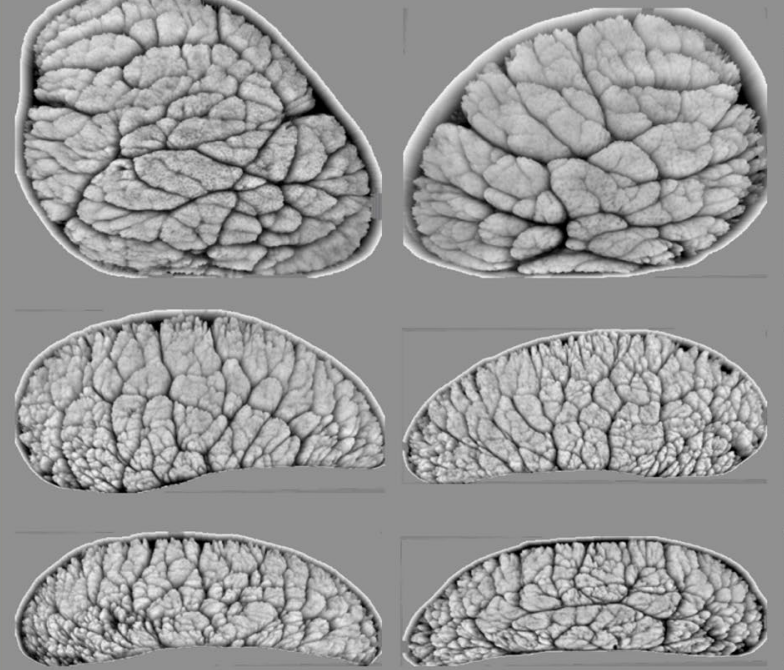

C
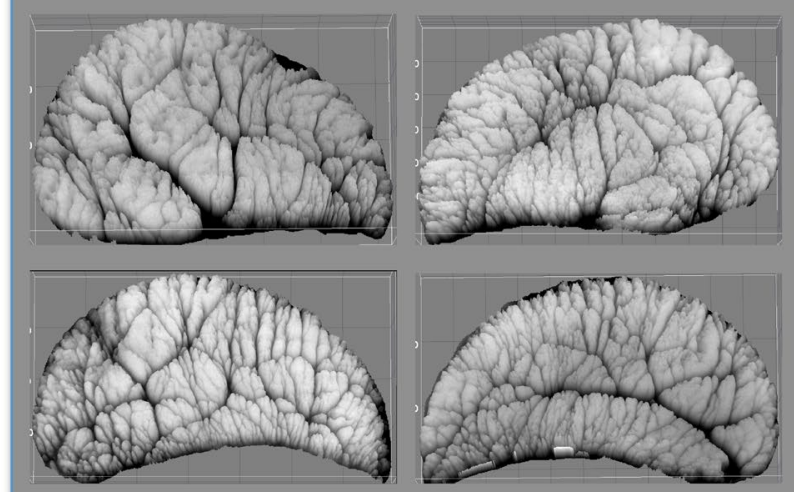

$\mathrm{m}$
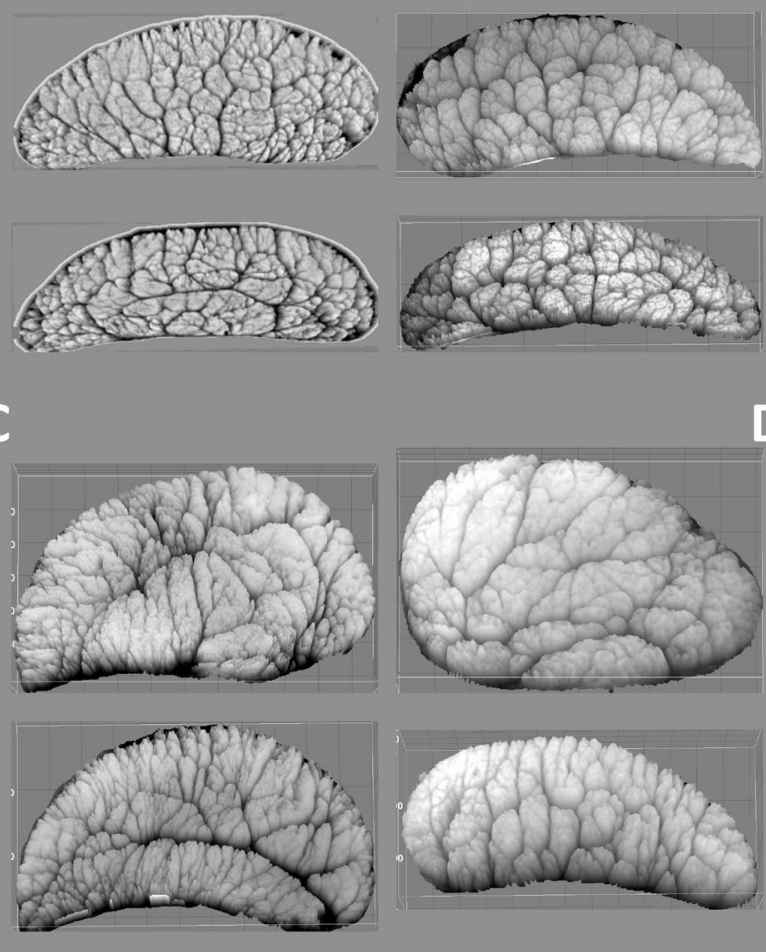

D
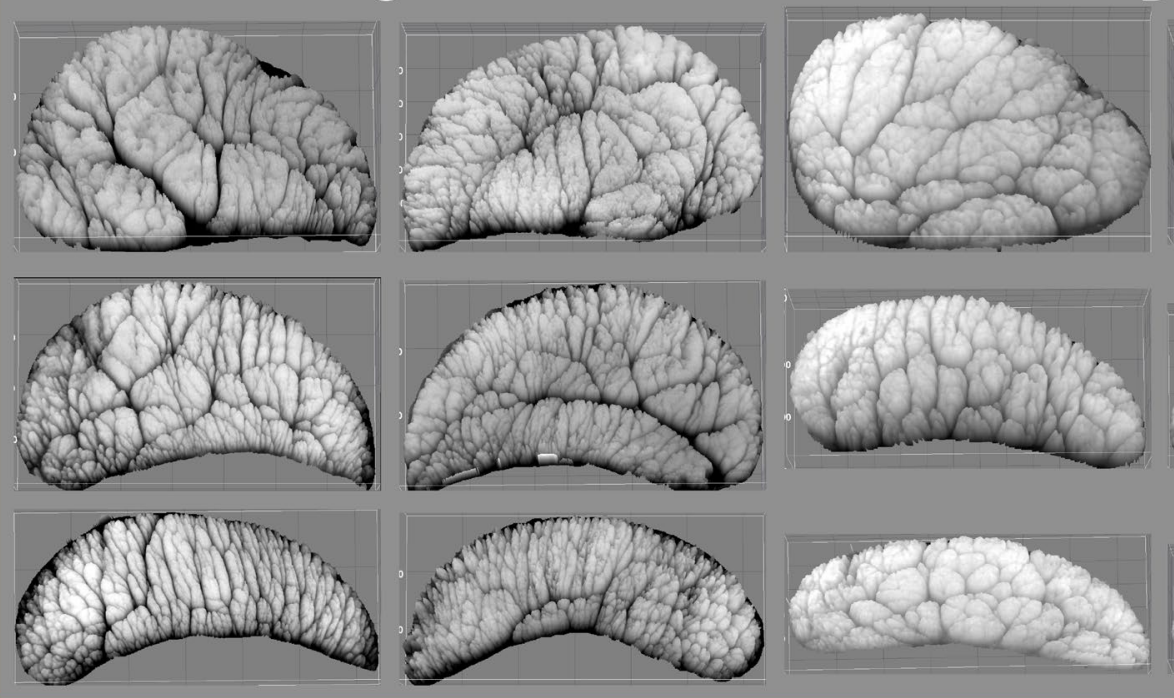
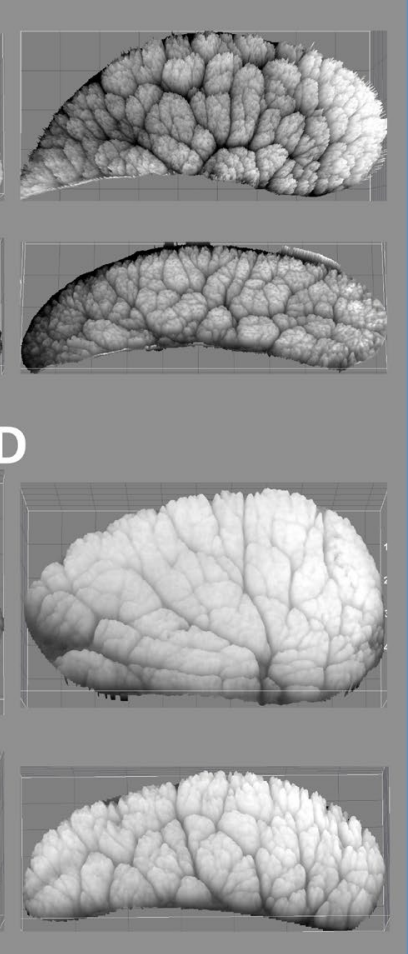

B

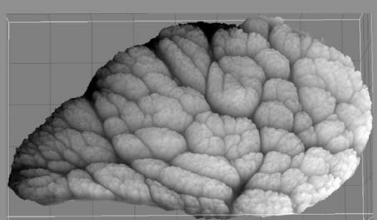

)

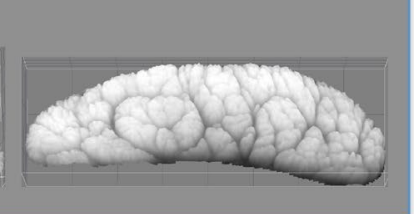

Fig. 3. Left-right asymmetry of fascicle organisation. Images of single tendon slices from the proximal (p), mid-metacarpal (m) and distal (d) regions of both the left (L) and right (R) legs at 4 (A), 6 (B), 18 (C) and 21 (D) years old. The fascicular structures are neither identical nor mirror images of each other, although some similarities can be seen. 

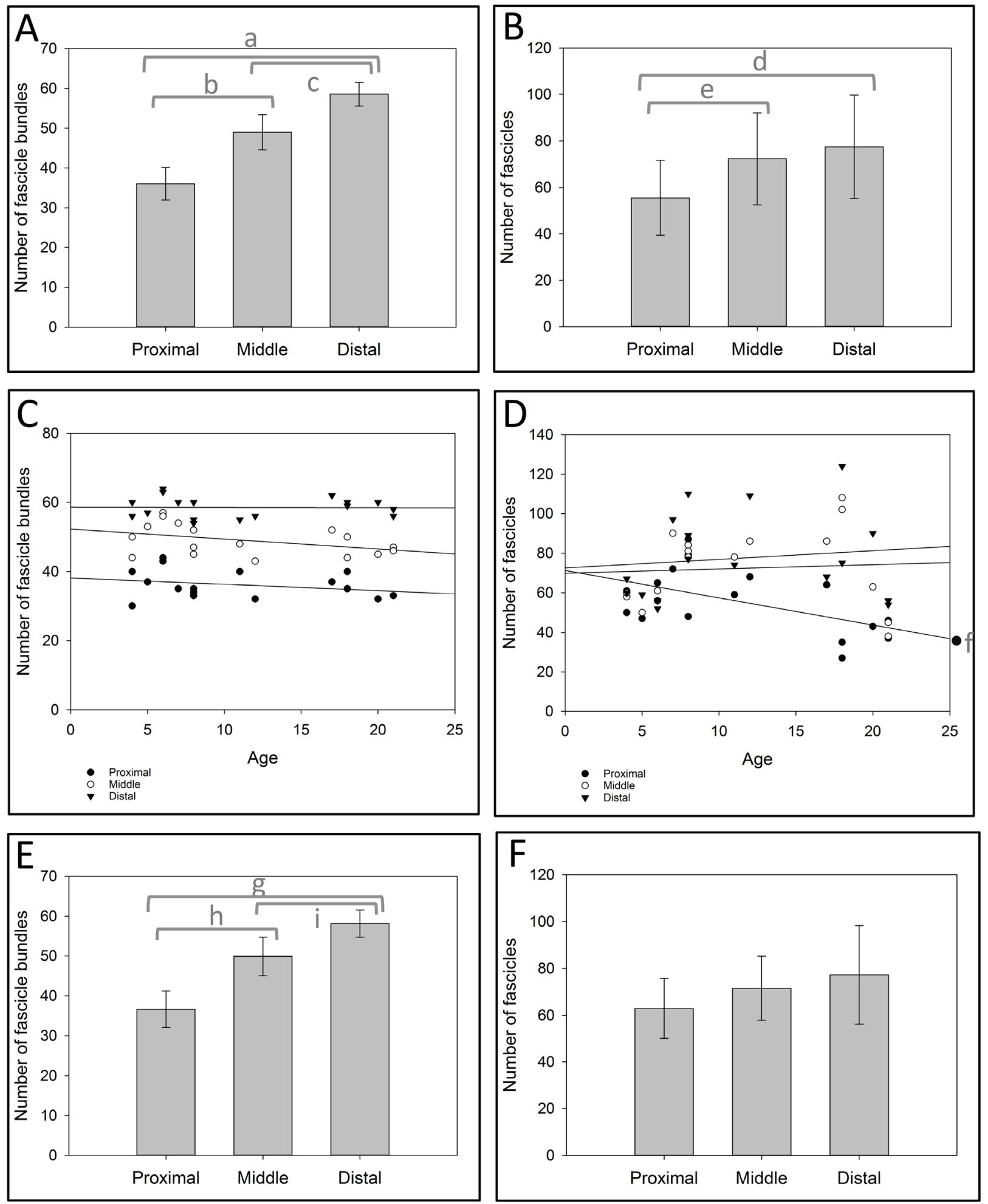

Fig. 4. Differences in fascicle numbers between regions and with age. A-B: Numbers of fascicle bundles (A) and fascicles $(\mathbf{B})$ in the proximal, middle and distal regions measured in 17 samples ranging from 4 to 21 years old. C-D: Plots of the number of fascicle bundles (C) and fascicles (D) in each region against age, for the samples analysed in A-B. E-F: Numbers of fascicle bundles (E) and fascicles (F) in the proximal, middle (midmetacarpal) and distal regions measured in 11 samples ranging from 4 to 12 years old. Data were analysed by one-way ANOVA with a Holm-Sidak post-hoc test (B, E \& F), Kruskal-Wallis with a post-hoc Tukey test (A) or by Pearson correlation (C-D). ${ }^{\mathrm{a}} p<0.001,{ }^{\mathrm{b}} p=0.002,{ }^{\mathrm{c}} p=0.006,{ }^{\mathrm{d}} p=0.006,{ }^{\mathrm{e}} p=0.031,{ }^{\mathrm{f}} p=0.0255 ; R=-0.539$, ${ }^{\mathrm{g}} p<0.001,{ }^{\mathrm{h}} p<0.001,{ }^{\mathrm{i}} p<0.001$. 
The number of fascicle bundles increased from the proximal to the distal end of the tendon and the number of fascicles decreased with age in the proximal region alone

The numbers of fascicles and fascicle bundles observed in images derived from whole slices of tendons ranging from 4 to 21 years old were recorded. The number of fascicle bundles increased from the proximal to the distal end of the tendon and the number of fascicles was lower in the proximal region (Fig. 4A-B). Furthermore, the number of fascicles, but not fascicle bundles, decreased with age in the proximal region (Fig. 4C-D). The fascicle numbers in each region were then reanalysed with the older ( $>15$ years) samples removed. The observed increase in fascicle bundle numbers from the proximal to the distal region was preserved (Fig. 4E) but the previously observed lower fascicle numbers in the proximal region were no longer present (Fig. 4F). The cross sectional area of 13 (of the 17) whole tendon slices ranging from 4 to 21 years old was determined
(Table 1). No statistically significant differences in tendon area were detected between regions or with age (not shown), despite alterations in tendon shape (Fig. 3) from the proximal to the distal region.

The mean thickness of the endotenon/IFM between fascicles decreased with age in the distal region

A histogram of all measurements of the endotenon/ IFM thickness in one forelimb SDFT from three eight-year-old horses displayed a single peak with a right-skew data distribution (Fig. 5A), indicating that the endotenon/IFM width between fascicles and fascicle bundles formed a broad continuum. The lack of a bimodal distribution indicated that endotenon/ IFM thickness could not be used as a discriminator between fascicles and fascicle bundles. However, superimposing the distributions for the fascicles and fascicle bundles indicated that the endotenon/ IFM was generally thicker between fascicle bundles than fascicles, and contained a wider range of
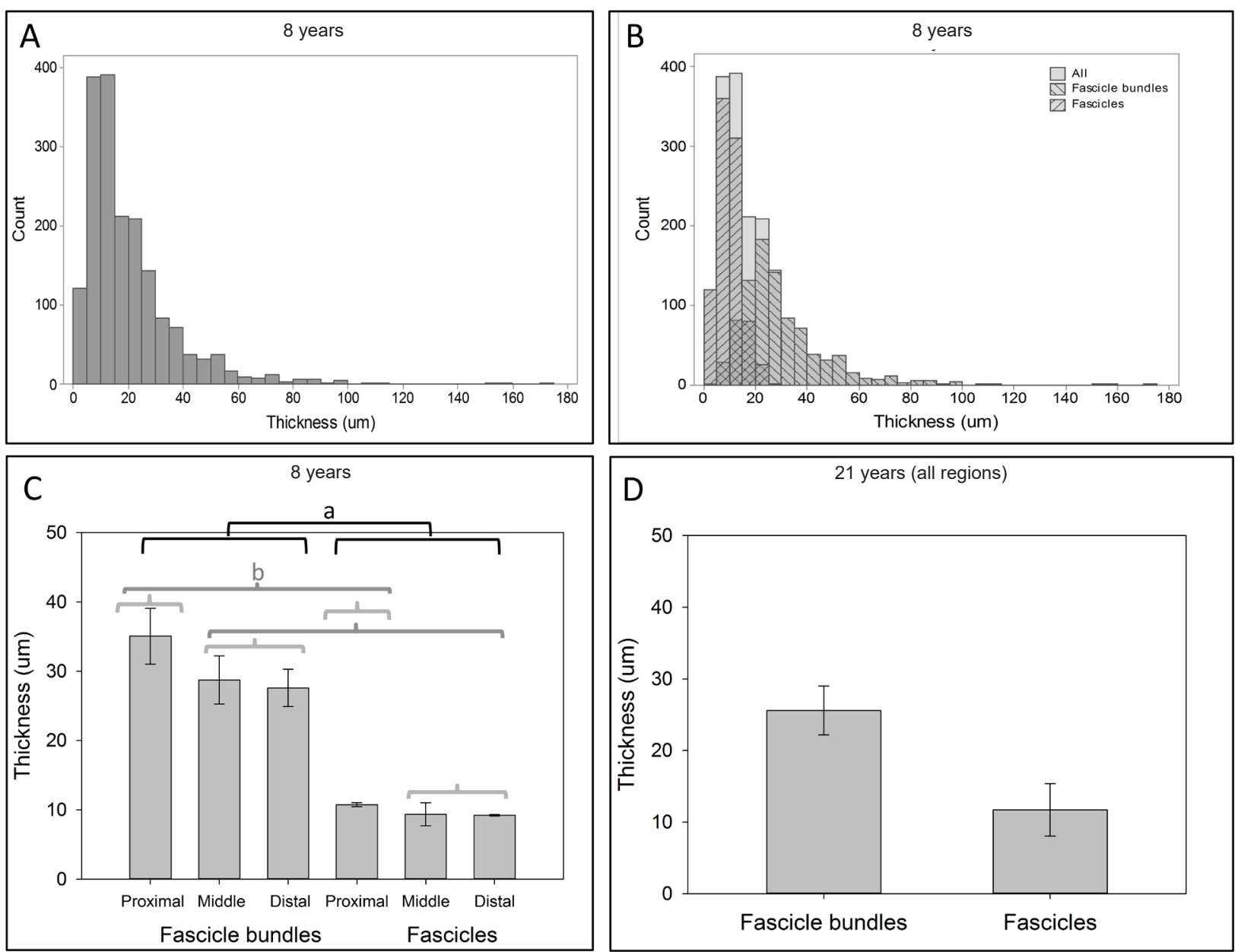

Fig. 5. Endotenon/IFM thickness in whole tissue slices. A: Histogram of endotenon/IFM thickness measurements derived from the proximal, mid-metacarpal and distal regions of three eight-year-old samples. B: Histogram of the data shown in A (all) with the distribution of fascicles and fascicle bundles superimposed. C: Bar chart of the mean endotenon/IFM thickness in each sample. D: Bar chart of the mean endotenon/IFM thickness in fascicles and fascicle bundles in the proximal, middle (mid-metacarpal) and distal regions of a single 21-year-old sample. ${ }^{\mathrm{a}} p<0.001,{ }^{\mathrm{b}} p=0.029$ (proximal versus distal) and 0.042 (proximal versus middle) by two-way ANOVA with a Holm-Sidak post-hoc test. 
larger thickness measurements (Fig. 5B). The mean thickness of the endotenon/IFM was greater in fascicle bundles than fascicles and in the proximal region by two-way ANOVA (Fig. 5C). Mean endotenon/ IFM thickness measurements were found to be approximately similar in a 21-year-old to those from an eight-year-old (Fig. 5D).

The endotendon/IFM width was then measured in sections from the proximal, mid-metacarpal and distal regions of the SDFT stained with H\&E, with 60 measurements taken per image. Again, the histogram showed that the endotenon/IFM was generally thicker between fascicle bundles than fascicles and contained a wider range of larger thickness measurements (Fig. 6A). The mean thickness of the endotenon/IFM was significantly greater in fascicle bundles than fascicles as well as in the proximal region as compared to the mid-metacarpal region, as assessed by one-way ANOVA (Fig. 6B). Two-way ANOVA was not carried out for this data-set as the data were not suitable for parametric analysis and non-parametric two-way ANOVA did not permit post-hoc testing. The mean thickness of the endotenon/IFM in fascicles, but not fascicle bundles, was found to decrease in the distal region with age (Fig. 6 C\&D) and there was a trend for a decrease in the mid-metacarpal region.
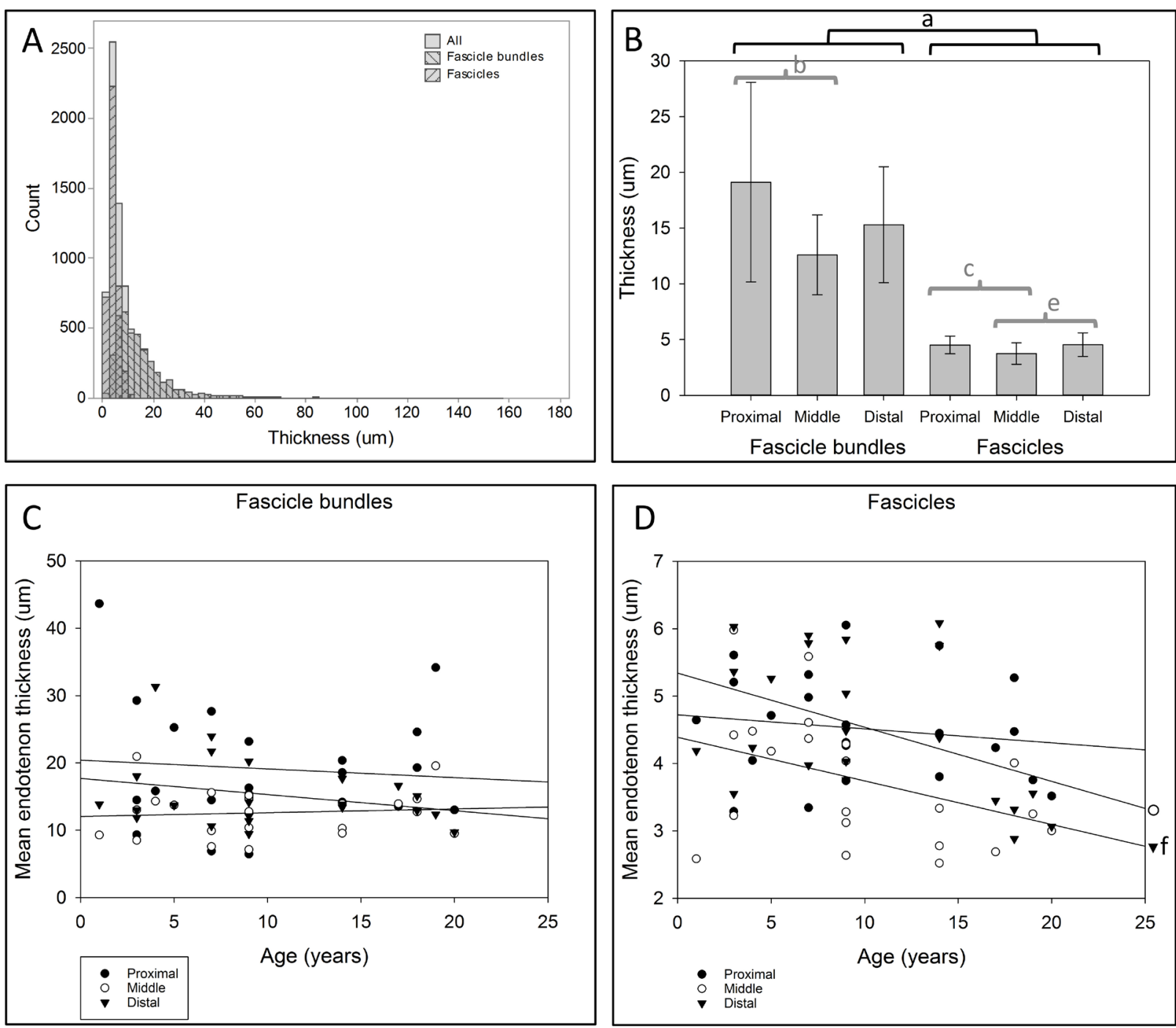

Fig. 6. Endotenon/IFM thickness in histological sections. A: Histogram of all endotenon/IFM thickness measurements with the underlying distribution of fascicles and fascicle bundles superimposed. B: Bar chart of the mean endotenon/IFM thickness between fascicles and fascicle bundles in the proximal, middle (midmetacarpal) and distal regions. C-D: Plots of the mean endotenon/IFM thickness in each region against age for fascicle bundles (C) and fascicles (D). All endotenon/IFM thickness measurements were derived from histological sections of the proximal, middle (mid-metacarpal) and distal regions of 22 tendon samples ranging from 1 to 20 years old. Data were analysed by one-way ANOVA considering fascicles and bundles and regional data for fascicles/bundles separately (B) or by Pearson correlation (C-D). ${ }^{a} p<0.001,{ }^{b} p=0.006$ (both Kruskal-Wallis with post-hoc Tukey test), ${ }^{c} p=0.017,{ }^{\mathrm{e}} p=0.002$ (both one-way ANOVA with post-hoc Holm-Sidak test), ${ }^{\mathrm{f}} p=0.0376 ; R=-0.446$. There was also a trend for a decrease of fascicles with age in the middle (mid-metacarpal) region; $p=0.0664 ; R=-0.398$. 

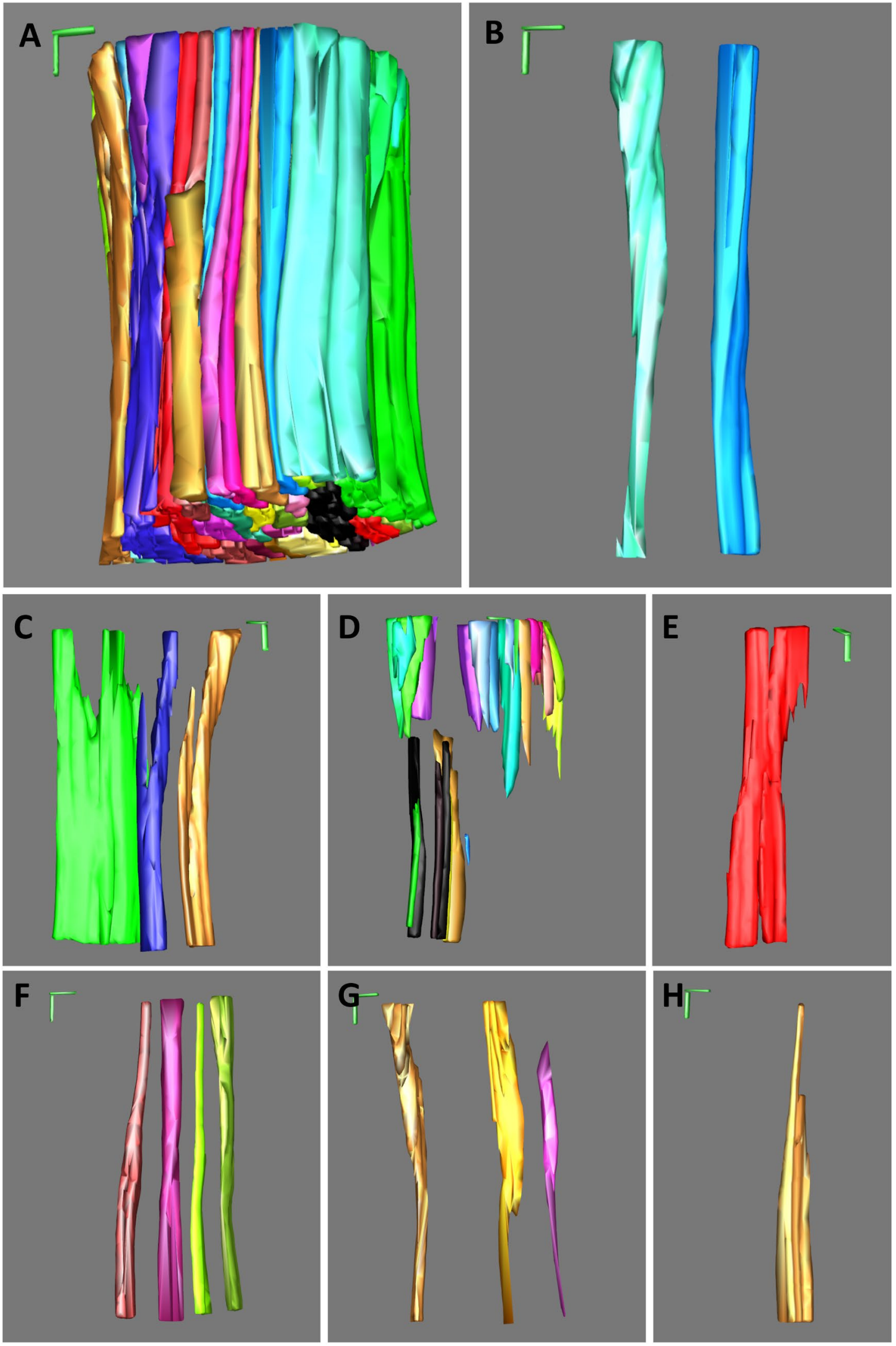

Fig. 7.3D reconstruction of an entire one-year-old tendon width in the mid-metacarpal region from histological sections. A: Entire reconstruction including all fascicles within a $1 \mathrm{~cm}$ length and $0.25 \mathrm{~cm}$ tissue width created using alternate sections from 500 serial $5 \mu \mathrm{m}$ sections. B-H: Selected fascicles from the 3D reconstruction. B: Two twisting fascicles. C: Three converging fascicles. D: Reconstructed fascicles not appearing to run the entire length of the 3D reconstruction and including one short fascicle entirely enclosed within the reconstruction (blue). E: Fascicle undergoing convergence and re-splitting. F: Four relatively straight fascicles. G: Three fascicles narrowing towards one end of the tissue block. H: One fascicle broadening towards the same end of the tissue block. Scale bar $=1 \mathrm{~mm}$. 
Fascicles had a range of morphologies and fascicular structure specified during foetal development

All fascicles within a $10 \mathrm{~mm}$ length and $2.5 \mathrm{~mm}$ width of SDFT from a one-year old horse were reconstructed (Fig. 7A; Video 2). Whilst several fascicles were present throughout the $10 \mathrm{~mm}$ length of the reconstruction (Fig. 7B, E-H), several fascicles appeared to terminate within the reconstruction. One short fascicle (blue) was entirely enclosed within the tissue volume (Fig. 7D). However, it could not be excluded that the shorter fascicles were laterally connected to other fascicles within the reconstruction. Three fascicles converged (Fig. 7C) and one fascicle demonstrated re-splitting (Fig. 7E). Whilst some fascicles were straight (Fig. 7F), others displayed

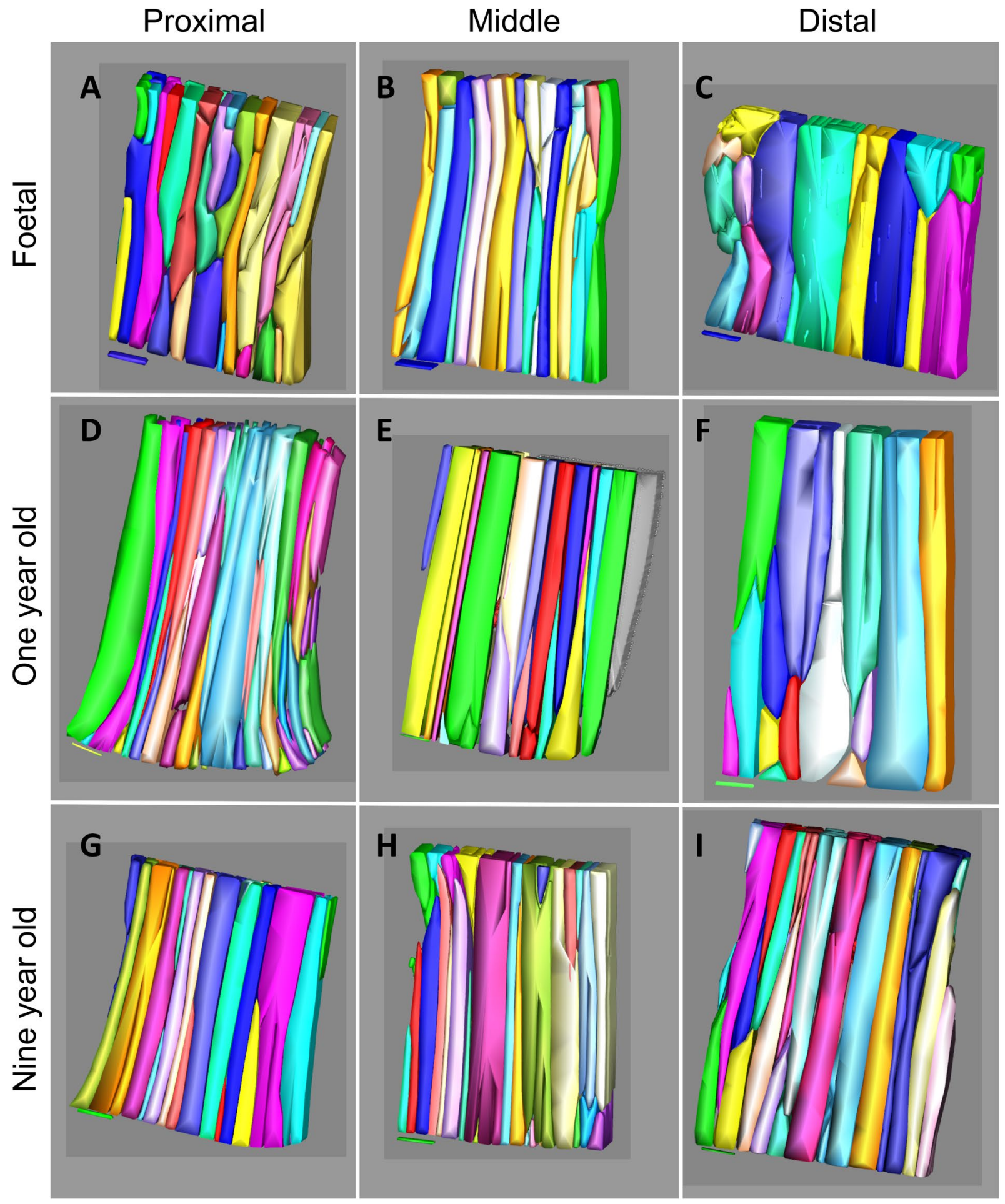

Fig. 8. 3D reconstruction of foetal (A-C), one-year-old (D-F) and nine-year-old (G-I) tendon from the proximal (A, D, G), mid-metacarpal (B, E, H) and distal (C, F, I) regions using histological sections. Each reconstruction was derived from a $10 \mathrm{~mm}$ length of tissue and from 20 sequential $5 \mu \mathrm{m}$ sections therefore encompassing an approximately $100 \mu \mathrm{m}$ tissue width. Scale bar $=1 \mathrm{~mm}$. 
evidence of internal twisting (Fig. 7B). Several fascicles either narrowed (Fig. 7G) or broadened (Fig. $7 \mathbf{H}$ ) from one end of the reconstruction to the other.

To determine whether fascicle morphology is specified during development, differs between regions and alters during tissue maturation, 3D reconstruction was carried out on serial histological sections from foetal, one-year-old and nine-year-old SDFT tissue from the proximal, mid-metacarpal and distal regions (Fig. 8). Reconstruction of foetal tissue from whole tendon slices was not possible due to the small size of the sample. The sample from the nineyear-old was chosen to represent a fully mature adult tendon. Reconstructions encompassed a $10 \mathrm{~mm}$ tissue length and a $100 \mu \mathrm{m}$ tissue width. Fascicles were arranged longitudinally in each reconstruction but fascicles did not always run the entire length of the reconstruction. There appeared to be wider fascicles at the distal end, particularly in younger tissue (Fig. $8 \mathbf{C}, \mathbf{F})$; however, a similar arrangement of fascicles was observed in all samples.

\section{Discussion}

Three-dimensional reconstruction of the structure of the endotenon/IFM and fascicles from whole tendon slices and using histological images indicated that equine SDFT fascicles were far from being disconnected continuous parallel structures. Fascicles were found to diverge, converge, undergo partial convergence and to re-split, whilst some fascicles did not continue as separate entities within the reconstructed volume (Figs. 2 \& 7). Whilst approximately cylindrical, fascicles adopted a variety of morphologies. It is reported that collagen fibres within tendon fascicles are not necessarily parallel and can cross or be plaited (Kannus, 2000). Interweaving fascicles (termed 'secondary') and fascicle bundles (termed 'tertiary') are also described, particularly fascicle bundles at the insertion point with the bone (Elliott, 1965), although other studies indicate that fascicles are largely separate entities within tendon (Edwards, 1946; Kastelic et al., 1978). It may be that the highly specialised energy-storing function of the high strain equine SDFT requires a complex interweaving arrangement of fascicles. Whilst some fascicles appeared to be discontinuous, lateral connections could not be entirely excluded and the suggested regions of discontinuity would not exclude the possibility that collagen fibrils could be continuous from one end of the tendon to the other (Provenzano and Vanderby, 2006; Svensson et al., 2017), as fibrils could transfer between fascicles as they interweave throughout the tendon. However, the presence of transversely-orientated endotenon/ IFM across several serial sections (Fig. 1C,D) indicated that many of the collagen fibrils abutting such regions would either terminate within the tissue, or become incorporated into and reorganised within the interfascicular matrix.
There was a lack of symmetry in the arrangement of fascicles between the left and right limbs, in different regions or at different ages, although there were some similarities in the location of the widest part of the endotenon/IFM adjacent to the epitenon (Fig. 3). The fascicular structure was clearly evident in a reconstructed foetal sample (Fig. 8). It may therefore be the case that the development of fascicular pattern is a stochastic process that begins at a particular point at the tendon periphery.

During reconstruction of fascicles from histological images it was noted that some, but not all, fascicles appeared to be twisted (Fig. 7). A helical arrangement of fascicles is reported in the equine SDFT (Thorpe et al., 2013a; Thorpe et al., 2014; Thorpe et al., 2015b), the human Achilles (Szaro et al., 2009) and extensor carpi ulnaris tendon (Kalson et al., 2012), bovine flexor and rat tail tendon (de Campos Vidal, 2003; Reese and Weiss, 2013), canine patellar tendon and anterior cruciate ligament (Yahia and Drouin, 1989). Collagen fibres (fibril bundles) in chick metatarsal tendon and mouse Achilles and tail tendon appear to define tendon crimp which governs the non-linearity of the tendon stress-strain curve (Kalson et al., 2015). However, fascicle twisting may contribute to energy preservation in cyclic loading which is important for efficient locomotion (Thorpe et al., 2013a). Therefore, in energy-storing tendons such as the equine SDFT, it is likely that the endotenon/IFM facilitates fascicle sliding (Thorpe et al., 2015a), fibril crimp protects against immediate force-transfer through fibrils, and the fascicular helices allow recoil and energy recovery. The three-dimensional interweaved nature of the fascicles and fascicle bundles within the equine SDFT may provide a mechanism to dampen longitudinal shearing within the endotendon/IFM during fascicle sliding and to distribute forces throughout the tendon. Nevertheless, the central core of the equine SDFT is more prone to injury and it may be that some shearing does occur in this region. It is currently unknown whether other energy-storing tendons have a similar arrangement of fascicles to the equine SDFT.

In the present study it was found that the thickness of the endotenon/IFM between fascicles was decreased with age in the distal region, with a trend for an agerelated decrease in the mid-metacarpal region. The endotenon/IFM was thinner between fascicles than fascicle bundles, for both whole tissue slices and histological sections, which was not an unexpected result given that apparent endotenon thickness was used as a defining parameter to categorise fascicles and fascicle bundles. However, endotenon thickness could not be used as a discriminator between fascicles and fascicle bundles, due to the overlap between the two sets of measurements (Fig. 5B \& 6A). The thickness of the endotenon/IFM measured in whole tissue slices (Fig. 5) was approximately one-third higher than that measured from histological images (Fig. 6). These differences could be attributed to differences in water content between the samples, 
with histological samples being dehydrated before embedding, whilst whole tissue slices were hydrated to aid visualisation of the endotenon/IFM structure. Tendons, from which whole tissue slices were derived, were also frozen before cutting; this could have resulted in an increased tissue cross-sectional area as well as alterations to the collagen fibril structure and biomechanical properties, as is known for tendons of other species (Giannini et al., 2008; Oswald et al., 2017). However, magnetic resonance imaging of horse limbs shows no differences in image quality between fresh and freeze-thawed samples, indicating that freeze-thawing may not affect the fascicular structure of tendon (Widmer et al., 1999). A previous study compares the percentage area occupied by endotenon/IFM in the mid-metacarpal region of young (3-8 years of age) and old (15-20 years) samples, imaged using scanning electron microscopy, and shows that it is smaller in older samples (Thorpe et al., 2013b). A reduced thickness of the endotenon/IFM, as also presented in the present study, could limit fascicle sliding, reduce tendon elasticity and increase damage to collagenous fascicles. Alternatively, a thinner endotenon/IFM could increase the possibility of shearing between fascicles and more readily produce gross tissue damage at this point. The presence of transversely orientated, often zigzagging, regions of endotendon/ IFM (Fig. 1C,D) could represent particular weak points within the tissue that would be increasingly susceptible to damage with ageing. A concentration of transversely orientated endotenon/IFM in particular areas, as indicated in the larger 3D reconstruction of fascicles from histological sections (Fig. 7D), may lead to site-specific damage that could rapidly propagate to produce an internal lesion. Protein turnover in the endotenon/IFM is decreased with age (Thorpe $e t$ al., 2016b) so potentially an accumulation of longer lived extracellular matrix proteins in the endotenon/ IFM could result in a denser and more compact tissue interface prone to damage.

An unexpected finding was that the number of fascicles decreased with age in the proximal region alone. This was probably not due to fascicles fusing, as a result of decreased endotenon/IFM thickness, because no age-related changes in endotenon thickness were observed in this region and no alteration in the number of fascicle bundles or tendon cross-sectional area was observed. This finding could be confounded by the inclusion of three older hind-limb samples, although other parameters were not affected, or potentially, whole fascicles could be resorbed or lost during ageing and compensated by increased fascicle size. Whilst, turnover rates of collagen are very low in the mid-metacarpal region of the equine SDFT (Thorpe et al., 2010b), this may not be the case in the proximal region. It was also found that the number of fascicle bundles increased from the proximal to the distal end of the tendon and this might result from fascicle bundle divergence, or from new bundles starting within or at the periphery of the tendon.

Understanding the structure of tendon is important to develop strategies for injury prevention and treatment and to devise exercise regimens that allow tendons to work within their biomechanical tolerances, particularly during ageing. Furthermore, recapitulation of the fascicular architecture of tendon is likely to be important for tissue engineering strategies that aim to generate biosynthetic tendon replacements. Our results indicated that engineering and repairing energy-storing tendons are likely to pose significant challenges due to complexity of the fascicular anatomy.

\section{Conclusions}

In conclusion we have shown that the equine SDFT has a complex three-dimensional fascicular architecture, with evidence of fascicular convergence and divergence leading to an interconnected and interweaved structure. Endotendon/IFM thickness and fascicle numbers were altered with age in specific regions, indicating that the effects of ageing are heterogeneous longitudinally in the tendon. The results presented may have wider applicability to energy-storing tendons in other species, including man.

\section{Acknowledgements}

O.J. Ali was supported by a PhD scholarship from the Iraqi Government's Higher Committee for Educational Development (HCED). The contribution of the Philip Leverhulme Equine Hospital staff, particularly T. Jopson, and the owners that gifted tissue for use in research is gratefully acknowledged.

\section{References}

Alexander RM (1991) Energy-saving mechanisms in walking and running. J Exp Biol 160: 55-69.

Birk DE, Trelstad RL (1986) Extracellular compartments in tendon morphogenesis: collagen fibril, bundle, and macroaggregate formation. J Cell Biol 103: 231-240.

Birk DE, Zycband EI, Winkelmann DA, Trelstad RL (1989) Collagen fibrillogenesis in situ: fibril segments are intermediates in matrix assembly. Proc Natl Acad Sci U S A 86: 4549-4553.

Canty EG, Lu Y, Meadows RS, Shaw MK, Holmes DF, Kadler KE (2004) Coalignment of plasma membrane channels and protrusions (fibripositors) specifies the parallelism of tendon. J Cell Biol 165: 553-563. 
Clayton RA, Court-Brown CM (2008) The epidemiology of musculoskeletal tendinous and ligamentous injuries. Injury 39: 1338-1344.

Craig AS, Birtles MJ, Conway JF, Parry DA (1989) An estimate of the mean length of collagen fibrils in rat tail-tendon as a function of age. Connect Tissue Res 19: 51-62.

de Campos Vidal B (2003) Image analysis of tendon helical superstructure using interference and polarized light microscopy. Micron 34: 423-432.

Edwards DAW (1946) The blood supply and lymphatic drainage of tendons. J Anat 80: 147-152.

Elliott DH (1965) Structure and function of mammalian tendon. Biol Rev Camb Philos Soc 40: 392-421.

Fallon J, Blevins FT, Vogel K, Trotter J (2002) Functional morphology of the supraspinatus tendon. J Orthop Res 20: 920-926.

Giannini S, Buda R, Di Caprio F, Agati P, Bigi A, De Pasquale V, Ruggeri A (2008) Effects of freezing on the biomechanical and structural properties of human posterior tibial tendons. Int Orthop 32: 145-151.

Handsfield GG, Slane LC, Screen HR (2016) Nomenclature of the tendon hierarchy: an overview of inconsistent terminology and a proposed sizebased naming scheme with terminology for multimuscle tendons. J Biomech 49: 3122-3124.

Hess GW (2010) Achilles tendon rupture: a review of etiology, population, anatomy, risk factors, and injury prevention. Foot Ankle Spec 3: 29-32.

Kalson NS, Lu Y, Taylor SH, Starborg T, Holmes DF, Kadler KE (2015) A structure-based extracellular matrix expansion mechanism of fibrous tissue growth. eLife 4: doi: 10.7554/eLife.05958.

Kalson NS, Malone PS, Bradley RS, Withers PJ, Lees VC (2012) Fibre bundles in the human extensor carpi ulnaris tendon are arranged in a spiral. J Hand Surg Eur Vol 37: 550-554.

Kannus P (2000) Structure of the tendon connective tissue. Scand J Med Sci Sports 10: 312-320.

Kasashima Y, Takahashi T, Smith RK, Goodship AE, Kuwano A, Ueno T, Hirano S (2004) Prevalence of superficial digital flexor tendonitis and suspensory desmitis in Japanese Thoroughbred flat racehorses in 1999. Equine Vet J 36: 346-350.

Kastelic J, Galeski A, Baer E (1978) The multicomposite structure of tendon. Connect Tissue Res 6: 11-23.

Kremer JR, Mastronarde DN, McIntosh JR (1996) Computer visualization of three-dimensional image data using IMOD. J Struct Biol 116: 71-76.

Maffulli N, Waterston SW, Squair J, Reaper J, Douglas AS (1999) Changing incidence of Achilles tendon rupture in Scotland: a 15-year study. Clin J Sport Med 9: 157-160.

Meghoufel A, Cloutier G, Crevier-Denoix N, de Guise JA (2010) Ultrasound B-scan image simulation, segmentation, and analysis of the equine tendon. Med Phys 37: 1038-1046.

Nyyssonen T, Luthje P, Kroger H (2008) The increasing incidence and difference in sex distribution of Achilles tendon rupture in Finland in 1987-1999. Scand J Surg 97: 272-275.

Oswald I, Rickert M, Bruggemann GP, Niehoff A, Fonseca Ulloa CA, Jahnke A (2017) The influence of cryopreservation and quick-freezing on the mechanical properties of tendons. J Biomech 64: 226230.

Perkins NR, Reid SW, Morris RS (2005) Risk factors for injury to the superficial digital flexor tendon and suspensory apparatus in Thoroughbred racehorses in New Zealand. N Z Vet J 53: 184-192.

Provenzano PP, Vanderby R, Jr. (2006) Collagen fibril morphology and organization: implications for force transmission in ligament and tendon. Matrix Biol 25: 71-84.

Reese SP, Weiss JA (2013) Tendon fascicles exhibit a linear correlation between Poisson's ratio and force during uniaxial stress relaxation. J Biomech Eng 135: 34501.

Russo V, Mauro A, Martelli A, Di Giacinto O, Di Marcantonio L, Nardinocchi D, Berardinelli P, Barboni B (2015) Cellular and molecular maturation in fetal and adult ovine calcaneal tendons. J Anat 226: 126-142.

Schneider CA, Rasband WS, Eliceiri KW (2012) NIH Image to ImageJ: 25 years of image analysis. Nat Methods 9: 671-675.

Svensson RB, Herchenhan A, Starborg T, Larsen M, Kadler KE, Qvortrup K, Magnusson SP (2017) Evidence of structurally continuous collagen fibrils in tendons. Acta Biomater 50: 293-301.

Szaro P, Witkowski G, Smigielski R, Krajewski P, Ciszek B (2009) Fascicles of the adult human Achilles tendon - an anatomical study. Ann Anat 191: 586-593.

Thorpe CT, Clegg PD, Birch HL (2010a) A review of tendon injury: why is the equine superficial digital flexor tendon most at risk? Equine Vet J 42: 174-180.

Thorpe CT, Godinho MS, Riley GP, Birch HL, Clegg PD, Screen HR (2015a) The interfascicular matrix enables fascicle sliding and recovery in tendon, and behaves more elastically in energy storing tendons. J Mech Behav Biomed Mater 52: 8594.

Thorpe CT, Karunaseelan KJ, Ng Chieng Hin J, Riley GP, Birch HL, Clegg PD, Screen HR (2016a) Distribution of proteins within different compartments of tendon varies according to tendon type. J Anat 229: 450-458.

Thorpe CT, Klemt C, Riley GP, Birch HL, Clegg PD, Screen HR (2013a) Helical sub-structures in energystoring tendons provide a possible mechanism for efficient energy storage and return. Acta Biomater 9: 7948-7956.

Thorpe CT, Peffers MJ, Simpson D, Halliwell E, Screen HR, Clegg PD (2016b) Anatomical heterogeneity of tendon: Fascicular and interfascicular tendon compartments have distinct proteomic composition. Sci Rep 6: 20455.

Thorpe CT, Riley GP, Birch HL, Clegg PD, Screen HR (2014) Fascicles from energy-storing tendons 
show an age-specific response to cyclic fatigue loading. J R Soc Interface 11: 20131058.

Thorpe CT, Spiesz EM, Chaudhry S, Screen HR, Clegg PD (2015b) Science in brief: recent advances into understanding tendon function and injury risk. Equine Vet J 47: 137-140.

Thorpe CT, Streeter I, Pinchbeck GL, Goodship AE, Clegg PD, Birch HL (2010b) Aspartic acid racemization and collagen degradation markers reveal an accumulation of damage in tendon collagen that is enhanced with aging. J Biol Chem 285: 1567415681.

Thorpe CT, Udeze CP, Birch HL, Clegg PD, Screen HR (2012) Specialization of tendon mechanical properties results from interfascicular differences. J R Soc Interface 9: 3108-3117.

Thorpe CT, Udeze CP, Birch HL, Clegg PD, Screen HR (2013b) Capacity for sliding between tendon fascicles decreases with ageing in injury prone equine tendons: a possible mechanism for age-related tendinopathy? Eur Cell Mater 25: 48-60.

Ward SR, Loren GJ, Lundberg S, Lieber RL (2006) High stiffness of human digital flexor tendons is suited for precise finger positional control. J Neurophysiol 96: 2815-2818.

Widmer WR, Buckwalter KA, Hill MA, Fessler JF, Ivancevich S (1999) A technique for magnetic resonance imaging of equine cadaver specimens. Vet Radiol Ultrasound 40: 10-14.

Yahia LH, Drouin G (1989) Microscopical investigation of canine anterior cruciate ligament and patellar tendon: collagen fascicle morphology and architecture. J Orthop Res 7: 243-251.

\section{Discussion with Reviewers}

Tom Shearer: Is it certain that fascicles converge, or is it possible that the gaps between them become smaller than the resolution of the images in places? Authors: The fascicles are separated by endotenon/ IFM although there is a tendency for gaps to be visible between fascicles in histological sections. When the endotenon/IFM becomes very thin between fascicles it is possible that it would not be visible in whole tissue sections; however, punctate white material is even visible within fascicles (Fig. 1A). Therefore, we consider that even thin endotenon/IFM would still be visible in whole tissue slices. Individual nuclei are visible in $H \& E$ stained histological sections; therefore, we consider that very thin endotenon/IFM would still be visible in histological images.

Tom Shearer: Observations were made regarding how endotenon thickness and fascicle numbers vary with age. Are there any data available on how the number of discontinuous fascicles varies with age? Authors: Close examination of serial sections indicated that lateral connections between fascicles could not be excluded even when transversely orientated endotenon/IFM, such as that shown in Fig. $1 \mathbf{C}, \mathbf{D}$, was clearly visible. Hence it was not possible to determine how the number of discontinuous fascicles may vary with age.

Valentina Russo: The end of a terminating fascicle could become a site of stress concentration. Did you observe any differences in the structures near the ends?

Authors: We did not observe any distortions to the fascicular architecture adjacent to transversely orientated endotenon/IFM; see for example Fig. 1C.

Valentina Russo: You describe both convergence and divergence of fascicles, do you believe that there is a directionality/chronology to how these structures are formed, i.e. starting out with many distinct fascicles which fuse, or alternatively starting out with few large bundles that subsequently split?

Authors: The endotenon/IFM was more extensive in the foetal sample, but the fascicular organisation appeared to be similar. Therefore, any fascicle fusion or bundle-splitting events are likely to have occurred prior to the ages analysed in this study. However, the apparent branching nature of the endotenon/IFM from the periphery to the core of the tendon indicated that an early fascicular template may be split by the developing endotenon/IFM.

Valentina Russo: When simply looking at a human patellar tendon for example, the fascicles visible on the surface usually appear to be continuous. Did you observe any systematic differences in fascicular organisation between the surface and the core of the horse SDF tendons?

Authors: In tissue slices, surface fascicles generally appeared to be more distinct; however, they could not be readily observed in whole tendons due to the thick epitenon.

Rene Svensson: Could the data obtained on tendon structure and microarchitecture be crucial to understand which strategies have to be undertaken to fabricate 3D hierarchical scaffolds, recreating the anisotropic architecture and cellularity of native tissues?

Authors: The endotendon/interfascicular matrix plays an important role in tendon biomechanics and functional specialisation. Therefore, the fascicular and interfascicular architecture of tendons should be a key consideration when designing bioengineered tissue replacements or scaffolds for tendon repair.

Editor's note: The Scientific Editor responsible for this paper was Juerg Gasser. 